\title{
Erratum: Transient birefringence of liquids induced by terahertz electric-field torque on permanent molecular dipoles
}

\author{
Mohsen Sajadi, Martin Wolf \& Tobias Kampfrath
}

Nature Communications 8:14963 doi: 10.1038/ncomms14963 (2017); Published 10 Apr 2017; Updated 25 May 2017

This Article contains typographical errors in six instances in which the convolution symbol '*' was mistakenly omitted from six equations during the production stage. Each instance is described below.

The penultimate sentence of the first paragraph of the Results subsection titled 'Model' should read:

'Therefore, $\Delta f_{1}$ does not yet cause optical birefringence but is accompanied by a time-dependent dielectric polarization $\mathbf{P}=\chi^{\mu_{0}} * \mathbf{E}$.'

The correct form of equation (3) is:

$$
\Delta n(t) \propto R_{2} *\left[E \cdot\left(N \Delta \alpha E+3 \chi^{\mu_{0}} * E\right)\right] .
$$

In the second paragraph after equation (3), the first sentence should read:

'Note that equation (3) reveals an analogy of the $\boldsymbol{\mu}_{\text {ind }}$ - and $\boldsymbol{\mu}_{0}$-related coupling mechanisms: the first field interaction generates an effective electronic $(N \Delta \alpha \mathbf{E})$ and orientational polarization $\left(\chi^{\mu_{0}} * E\right)$ which, in turn, serves as a handle for the second field interaction to generate a $P_{2}$-like perturbation (square bracket in equation (3)).'

In the paragraph preceding equation (11), the second sentence should read:

'This polarization is usually expressed by the convolution $P=\chi^{\mu_{0}} * E$ where $\chi^{\mu_{0}}$ is the contribution of the permanent electric dipole moment $\boldsymbol{\mu}_{0}$ to the familiar total dielectric susceptibility $\chi$.'

The correct form of equation (11) is:

$$
\Delta f_{1}(u, t) \propto P_{1}(u) \cdot\left(\chi^{\mu_{0}} * E\right)(t)
$$

The correct form of equation (12) is:

$$
\Delta f_{2}(u, t) \propto P_{2}(u) \cdot\left\{R_{2} *\left[E \cdot N \Delta \alpha E+3 E \cdot\left(\chi^{\mu_{0}} * E\right)\right]\right\}(t)
$$

Open Access This article is licensed under a Creative Commons Attribution 4.0 International License, which permits use, sharing, adaptation, distribution and reproduction in any medium or format, as long as you give appropriate credit to the original author(s) and the source, provide a link to the Creative Commons license, and indicate if changes were made. The images or other third party material in this article are included in the article's Creative Commons license, unless indicated otherwise in a credit line to the material. If material is not included in the article's Creative Commons license and your intended use is not permitted by statutory regulation or exceeds the permitted use, you will need to obtain permission directly from the copyright holder. To view a copy of this license, visit http://creativecommons.org/licenses/by/4.0/

(C) The Author(s) 2017 